\title{
Hybrid Service Recovery: Design for Seamless Inquiry Handovers between Conversational Agents and Human Service Agents
}

\author{
Mathis Poser \\ University of Hamburg \\ poser@informatik.uni-hamburg.de
}

\author{
Sukhpreet Singh \\ University of Hamburg \\ 3ssingh@informatik.uni-hamburg.de
}

\author{
Eva A. C. Bittner \\ University of Hamburg \\ bittner@informatik.uni-hamburg.de
}

\begin{abstract}
The effort of companies to deploy conversational agents (CAs) for customer self-service has been renewed due to their recent technological improvements. Despite their efficiency in processing recurring simple customer inquiries, limited capabilities of CAs to handle complex inquiries still lead to service failure and unsatisfied customers. Therefore, we propose a hybrid service recovery strategy with real-time handovers of inquiries from CAs to human service agents (HSAs), if CAs' capabilities are exceeded. Following a Design Science Research (DSR) approach, we present design principles (DPs) for the inquiry handover scenario, based on meta-requirements (MRs) derived from literature and expert interviews. By evaluating the DPs via prototype instantiation and process modulation, the suitability and interdependence of CAs' information collection activities and information presentation for handover could be verified.
\end{abstract}

\section{Introduction}

As customer satisfaction depends on service delivery features such as availability and accessibility, organizations continuously generate service innovations to meet customers' high expectations in terms of service quality $[1,2]$. With advancing technology, customer self-service has created opportunities to make service processes more efficient, save costs with reduced manual work and offer support at customers' convenience $[1,3]$. Recent improvements in artificial intelligence, especially in machine learning (ML), have revived companies' efforts to adopt conversational agents (CAs), e.g. chatbots, to elevate the intuition, richness and simplicity of self-service interactions [4]. CAs' capability to mimic human-to-human communication by autonomously interacting with humans via natural language $[5,6]$ constitutes an effective means to quickly provide engaging customer service irrespective of manual service operating hours [7].
Despite their potential to improve availability and accessibility, CAs are still bounded in handling customer inquiries [8, 9]. That is, CAs' capabilities are mostly restricted to retrieving fact-based, predefined response types (e.g. FAQs) and processing recurring data-intensive requests (e.g. change pickup location for package) [10-12]. Requests that are more complex (e.g. how or why questions) or linguistically ambiguous often exceed CAs' problem-solving or language understanding capacity, could trap customers in conversation loops and leave them unsatisfied with an unsolved problem [13]. This experience may frustrate customers' expectations toward chat-based self-service and lead to drop outs in the service process $[11,14]$.

Thus, scholars call for service recovery strategies to compensate technological boundaries during service delivery and avoid customer dissatisfaction [1517]. In this matter, previous research has focused on CA-initiated repair strategies to avoid conversational breakdown, ensure continuation of the dialog process and successful outcome $[18,19]$. Nevertheless, repair attempts can fail repeatedly, customers' requests can be too complex for CAs to offer help or require handling by human service agents (HSAs) due to company policies (e.g. reimbursements) [11,20]. Consequently, established fallback mechanisms that involve escalation of requests to HSAs are relevant to avoid complete service failures. However, to facilitate positive customer experience and meet customers' desire for short resolution time, approaches are required to assure real-time request processing by a HSA [19]. Therefore, instant chat-based handovers from automatic to manual processing are crucial to recover from CA failure. To realize this hybrid service recovery strategy, the design has to consider the socio-technical interplay of technology, processes and humans (employees and customers) and goes beyond technical system specifications [21-23]. To prevent customer frustration and support HSAs, relevant information of the CA-customer-interaction should be provided for the handover so that service process steps and questions do not have to be repeated [13, 17]. Therefore, 
connected processes are required to promote seamless handovers from CAs to HSAs [24, 25]. Moreover, CAs have to be configured to systematically collect information to present interim results to HSAs in a comprehensible format [25]. Hence, the goal of designing real-time inquiry handover as a CA service recovery strategy is addressed with the following research question: How to design the point of customer inquiry handover between CAs and HSAs?

The paper is structured as follows: Section 2 addresses the state of literature regarding CAs and customer self-service. Subsequently, the applied Design Science Research (DSR) approach is outlined. In Section 4, design principles (DPs) are presented, based on meta-requirements (MRs) from literature and expert knowledge. Section 5 presents the results from expert interviews to evaluate a DP-based mixed-fidelity prototype and process model. Results, contributions and limitations of the paper are discussed in Section 6 .

\section{Related work}

\subsection{Conversational agents}

CAs are software systems, which interact with humans via natural language (voice, text or both) [6, 26, 27]. Several terms (e.g. chatbot, dialog system, cognitive assistant) are used to refer to CAs with different communication modes, representations and application contexts (general-purpose vs. domainspecific) [26, 28]. Since the initial CA (ELIZA) [29], technological advancements in ML and natural language processing (NLP) have led to a significant expansion of CAs' capabilities [30]. In customer service, CAs are primarily used to scale the provision of efficient assistance (e.g. complaint management) and information (e.g. for products) $24 / 7$ to customers in different contexts $[10,31]$. As such, chatbots are increasingly used as a text-based customer-facing channel for service delivery $[32,33]$. However, due to the complexity of natural language conversations and their basic model of human interlocutors, CAs still have shortcomings in responding to a wide range of topics and complex inquiries [13, 34]. Furthermore, customers' high expectations toward CAs' conversational capabilities lead to breakdowns [35]. These problems are related to deficiencies of the natural language understanding and dialog management components, which interpret input wrongly, hinder dialog process (intent and/or entity detection) and prevent information retrieval or action execution [19]. As a result, customer requests are misinterpreted, answered inappropriately (false positive) or issues remain unanswered (false negative) [36]. Thus, a large number of requests needs to be directly or eventually escalated to employees [11].

In research, different approaches exist to overcome CAs' technical boundaries. On the one hand, users are prompted to engage in conversational repair activities by replying to CAs' uncertainty expressions (with or without alternatives) or rephrasing their input [18, 20]. On the other hand, employees are involved to avoid breakdowns by selecting an appropriate answer from CAs' suggestions [12, 37]. Nevertheless, existing approaches do not yet provide effective strategies for escalating requests to HSAs, (1) for which repeated repair attempts have failed or (2) which require employee handling as an (interim) result of a conversation. For such cases, an adaption of fallback solutions is needed where requests are deferred to employees. To avoid negative effects in terms of customer dissatisfaction due to additional waiting times, these solutions should address the challenge of realtime support [19]. The seamless handover of requests from CAs to HSAs as a recovery strategy addresses the suggestion in the literature of transferring requests to employees, if CAs' capacities are exceeded [12, 17 , $32,38]$.

\subsection{Customer (self-)service}

Companies aim to provide satisfying high quality service to customers, while increasing the efficiency and cost-effectiveness of service delivery. To ensure this, the design of the nature of daily service encounters with customers is of particular relevance to companies as they affect customer loyalty and consequently firm profitability [39]. In the past, service encounters were restricted to direct dyadic interactions between customers and employees, which are characterized by personalized and flexible service delivery with immediate feedback and elements of emotionality $[39,40]$. With evolving technology, service innovations have increased the range of service interfaces allowing companies to interact with customers through HSAs, technology or a combination of both (e.g. webpages, email, chat) $[1,41,42]$. This development has led to a successive transformation of customer-company interactions from personal and dialogbased to automated self-service [43]. Despite the advantages of accessibility and availability, self-service technologies are more standardized, less personalized and less interactive compared to personal service channels, which hamper value creation and customer experience [3, 40]. To increase the effectiveness of technology-infused self-service encounters in terms of customer experience, service providers increasingly deploy intelligent technology [8, 33]. With CAs' capabilities to conduct intuitive human-like conver- 
sations, to elicit feelings of social presence, empathy and personalization in customers, companies become able to emulate the beneficial characteristics of personal service encounters in self-service solutions [9, 28, 44]. In addition, CA technology has enabled companies to make service more efficient by reducing the number of routine requests that need to be handled by HSAs, while increasing the convenience of service delivery for customers $[3,33,40]$.

To fully take advantage of CAs and ensure high quality service, scholars call to investigate conditions, implications of and recovery strategies for service failure [15-17]. This knowledge is especially important for the prevention of detrimental effects connected to possible failures by CAs (e.g. for complex inquiries), as customers' satisfaction for service encounters with self-service technology inter alia depends on effective service recovery [45]. In this regard, the involvement of HSAs can ensure customer retention and avoid the abonnement of the self-service channel [14]. Therefore, to realize an effective CA service recovery strategy, service processes are required that enable the integration of multiple service interfaces [41]. Moreover, as satisfaction with the outcome of service recovery depends on interactionand process-related factors [46, 47], a well-designed process for the request handover is required to avoid repetitive service delivery steps. This design should promote effective and efficient processing by HSAs, who take over inquiries from a $\mathrm{CA}$ in real-time.

\section{Methodology}

This paper presents a DSR project, which is structured in accordance to Hevner's [48] three cycle view (see Figure 1). By starting the relevance cycle, a relevant real-world problem is identified that pertains to the improvement of repair strategies involving request escalation to HSAs to avoid CAs' service failures (Section 1 \& 2, step 1). The derivation of MRs for the design of and process for the customer inquiry handover is achieved, on the one hand, by considering domain specific knowledge of six experts with semistructured interviews (Section 4.2., step 2) [49]. The interviewees (I) (age: 26-35; male: 4; female: 2), have experience in handling product-, service-, or technology-related inquiries from external customers and/or developing chatbot systems for the customer service of different organizations. For the semistructured interviews, a guideline with three thematic categories was developed: (1) work and service processes, (2) CAs in service and (3) hybrid service. The qualitative content analysis of the transcribed interviews, which lasted 42 minutes on average, was conducted with MAXQDA software according to
Mayring [50]. The iterative and open coding approach was performed deductively and inductively by defining (sub-)categories and corresponding coding rules referring to literature (e.g. initial service encounter, service recovery) and transcript content (e.g. information gathering, timing of service recovery). On the other hand, in the rigor cycle, MRs

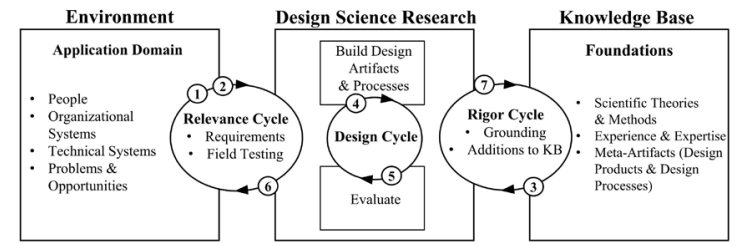

Figure 1. DSR three cycle view [48]

are derived from scientific literature (Section 4.1., step 3 ). In the design cycle, action and materiality-oriented DPs according to Chandra et al. [51] addressing the MRs are utilized to instantiate a mixed-fidelity prototype and a BPMN-based service process for the handover (Section 5.1., step 4). The two instantiations are demonstrated to five experts (female: 2; male: 3 ; age: 25-32) with experience in processing product-, service-, or technology-related inquiries as an external customer-facing service channel of different organizations (Section 5.1., step 5). By presenting both instantiations to the experts, the validity of implemented DPs was assessed by focusing on (1) the depicted CA's approach and the information presentation to assist HSAs in taking over an inquiry as well as (2) the process step sequence. For the evaluation (Section 5.2., step 6), semi-structured interviews were conducted with the same group of experts [49]. The interviews lasted 28 minutes on average and covered questions on the utility and suitability of the presented instantiations and underlying DPs. The content analysis was performed with a deductive coding approach utilizing MAXQDA [50]. The rigor cycle (step 7) is closed by adding prescriptive knowledge of form and function to literature, which contribute to a "theory of design and action" (Section 6) [52].

\section{Design requirements and principles}

Customers' evaluation of the outcome of a service recovery (e.g. rectification) depends on interactional and procedural factors $[46,47]$. These factors encompass communication with and treatment of customers (interactional) as well as process execution for the recovery (procedural) [47]. The identification of relevant insights from theory and practice was structured with the (1) initial service encounter and (2) service recovery phase regarding interactional and procedural factors [46]. As a result, a set of MRs, constituting the 
basis for the DPs, was derived by drawing on literature (L-MR) and practical knowledge (P-MR) from experts in the field of customer service operations.

\subsection{Meta-requirements from literature}

Initial service encounter: For the service encounter, customer satisfaction with technology-infused self-service channels depends on the responses to their needs and requests as well as the avoidance of systems' unprompted actions $[45,53]$. Thus, CAs should understand intentions or problems of customers to provide suitable assistance or solutions (L-MR1.1) [18, 32]. The identification and handling of inquiries should be addressed by maintaining a conversation with the customer that has the character of a natural dialogue (L-MR1.2) [31, 54]. During this conversation, the CA needs to be capable of interpreting customers' intentions by considering both the individual messages and the overall interaction context (LMR1.3) [55]. In addition, the CA should prompt customers with questions to provide more details, if the inquiry is missing relevant information (L-MR1.4) $[20,56]$. To converse via natural language, CAs need robust NLP capacity to automatically analyze input and generate adequate answers [5, 11, 19] (L-MR1.5). L-MR1: CA needs to interpret messages and interaction context to understand the inquiry, while conversing with a customer.

Service recovery: For service recovery, customer satisfaction depends on the response to a service failure $[45,53]$. Therefore, the CA should actively initiate handovers, if an inquiry exceeds the linguistic or problem-solving capabilities during customer interaction (L-MR2.1) [20, 38]. The CA needs to be aware of the service delivery process and monitor the status in order to anticipate service failure incidents (LMR2.2) [13]. L-MR2: CA needs to respond to a failure by actively initiating the handover. CAs deliver service in real-time, which means that the reason for a service failure cannot be analyzed prior to the incident [57]. Accordingly, the CA should identify relevant information during customer interaction to make corresponding data entries accessible from business applications (e.g. on product or customer) [28, 58] after the handover (L-MR3.2). L-MR3: CA needs to identify supplementary data available from business applications for the recovery.

\subsection{Meta-requirements from expert interviews}

Initial service encounter: During the initial service encounter, an important perquisite for CAs constitutes the preparation of the handover by systematically gathering information (I1, I3). In this context, the CA should attempt to comprehend and categorize the concern or question of the customer during the conversation to determine further steps (I1, I3, I4, I6) (P-MR1.1). Furthermore, CAs' capability to understand the context in a longer conversation is fundamental (I3, I5, I6) (P-MR1.2), since customers require varying time amounts to formulate the core of their request (I2). P-MR1: CA needs to comprehend and categorize an inquiry by capturing the conversational context. The documentation of inappropriate or incorrect information that makes further processing by HSAs cumbersome must be avoided (I1, I3). Thus, the CA should differentiate between valuable and irrelevant content (I4) (P-MR2.1). Accordingly, the CA should actively pose a set of relevant questions (I5). If there are ambiguities, follow-up questions need to be asked. The questions should be precise and their intentions have to be transparent to the customer to receive applicable information (I1, I6) (P-MR2.2). P-MR2: CA needs to pose initial and follow-up questions for ambiguous input and convey their intention to determine relevant information. The CA should encourage customers to describe the problem to determine its content (I3) (PMR3.1). Doing this, CAs' behavior should be characterized by polite and goal-directed behavior (no double questions) (I5), to maintain customers' satisfaction and willingness to cooperate (I1, I2) (PMR2.3). P-MR3: CA needs to act politely and goal determined to maintain customer's satisfaction.

Service recovery: In general, a CA should enable customers to forward their requests to a HSA at any time during the interaction (I1, I2, I3, I4, I6) (PMR4.1). In addition, a complete abortion caused by CAs' technical boundaries should be prevented to offer handovers as a service recovery (I6) (P-MR4.2). P-MR4: CA should offer handover options throughout interaction and prevent customer abortion before handover. The initiation of the recovery by the CA should base on different parameters. The inquiry handover should be introduced by the CA, if a certain amount of time (I2, I4) or a maximum number of failed attempts in the form of questions or propositions (e.g. three (I1, I5) has been reached (I3, I4, I5, I6) (PMR5.1). Therefore, the CA needs to register malfunctioning conversations caused by misguiding questions and unfitting solution proposals (I2, I4, I6) (P-MR5.2). P-MR5: CA needs to register misleading questions and solution proposals to initiate handovers adhering to defined limits of time and/or unsuitable propositions. A HSA should be forewarned by the system to prepare the continuation of the inquiry processing to realize an efficient real-time handover without delays 


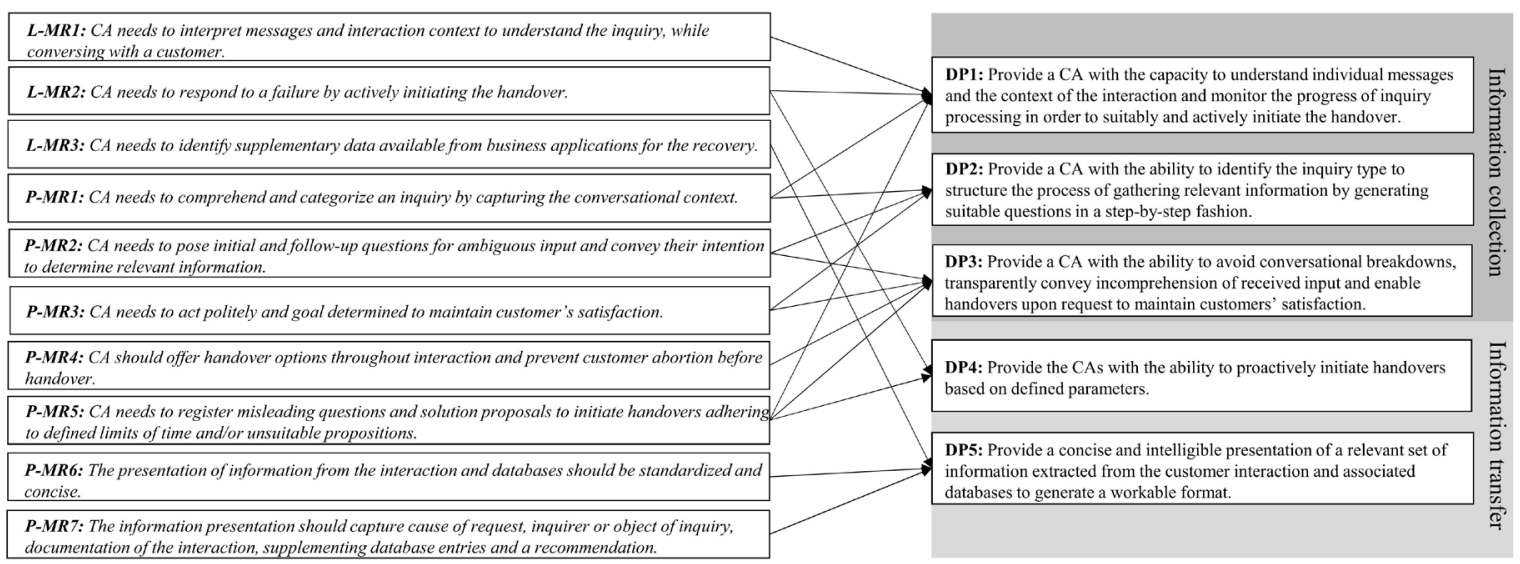

Figure 2. Meta-requirements (MR) and derived design principles (DP)

(I3, I6). Once a recovery has to be initiated by the CA, essential and available information extracted from the interaction between $\mathrm{CA}$ and customer should be compiled for the HSA (I1, I3) (P-MR6.1). In addition, the CA should verify and supplement information from the conversation by accessing databases (I4, I6) (P-MR6.2). The arrangement of visualized information needs to be standardized and concise so that HSAs can see the core elements of the request at a glance - in resemblance to a ticket system (I1, I3) (P-MR6.3). P-MR6: The presentation of information from the interaction and databases should be standardized and concise. For a workable information presentation, the determined cause of the request needs to be provided (e.g. problem/complaint, product order) (I1, I3, I5) (P-MR7.1). Furthermore, the identification of the inquirer and/or object of inquiry should be presented (I1, I3, I6) (P-MR7.2). In addition, details referring to solution attempts by the CA (I1, I2), documentation and short description of the conversation (I1) as well as a recommendation for a solution (I2, I4) should be generated. Moreover, applicable data from business applications, such as known errors and past decisions (I3, I4, I5), should be included in the inquiry summary (P-MR7.3). P-MR7: The information presentation should capture cause of request, inquirer or object of inquiry, documentation of the interaction, supplementing database entries and recommendation.

\subsection{Design principles for inquiry handovers}

Based on the MRs, five DPs are defined. The DPs are of the types form and function with substantial properties, capturing prescriptive knowledge to generate solutions for a hybrid service recovery strategy with real-time handovers from CAs to HSAs [59]. Following a supportive research approach [60], ten MRs were elicited. Three MRs emerged through knowledge from literature, seven MRs were obtained through interviews. The MRs and corresponding DPs are organized according to two categories, which emerged through the deductive-inductive coding process: (1) information collection and (2) information transfer (see Figure 2).

Information collection: The collection of information is a prerequisite for efficient inquiry handovers. Thus, the CA should be capable of tracking the inquiry processing progress and understanding individual messages and the context of the interaction to initiate the transfer in a suitable moment (DP1). HSAs require an information basis to continue the processing of a transferred inquiry in real-time. Thus, to gather relevant content and avoid the transfer of incorrect information, the interaction between customer and CA should be sustained by the ability of the CA to categorize the inquiry type (e.g. complaint) to subsequently generate suitable questions stepby-step (DP2). To avoid service failure during the initial service encounter, the CA should be able to avoid conversational breakdowns before inquiry handover and maintain customers' satisfaction and willingness to cooperate by transparently revealing incomprehension of input as well as enabling handovers upon customer request throughout the process (DP3).

Information transfer: The prevention of service failures requires the capability of a CA to proactively initiate inquiry handovers to a HSA, if technological boundaries have been reached. This handover initiation should base on adequate predefined parameters (DP4). For the service recovery by HSAs in real-time, the presentation of relevant information is a fundamental prerequisite. This information should be extracted, both, from the interaction with the customer and suitable databases. The presentation needs to be clearly structured and in a workable format (DP5). 


\section{Evaluation}

\subsection{Demonstration}

The demonstration of the instantiated DPs was achieved with a mixed-fidelity prototype and a BPMN-based process depiction addressing interactional and procedural aspects of the inquiry handover. The web-based proof-of-concept realized with HTML, CSS and JavaScript shows a script-based and chronological sequence of an interaction between customer and CA. The exemplary dialog was conceptualized with experts' descriptions of complaint inquiries, which exceed CAs' problem-solving capabilities. The prototype displayed messages in a chat window (Figure 3 left side for an excerpt) beginning with the first contact and ending with the initiation of a handover to a HSA (DP3 \& 4). In addition to the con-

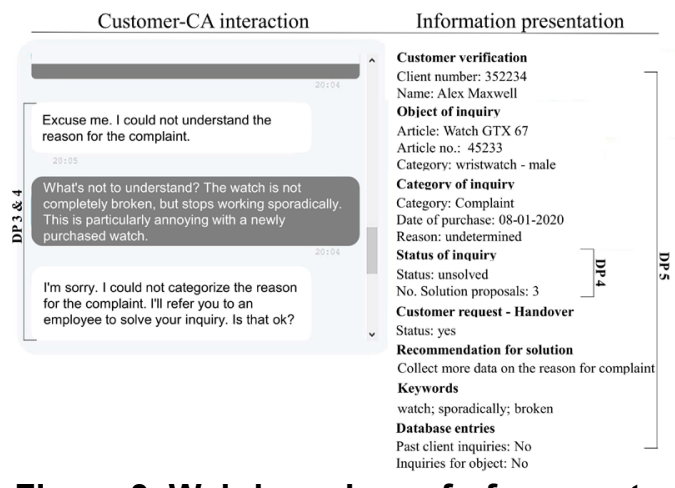

Figure 3. Web-based proof-of-concept

versational sequence, compiled information for the handover, gathered from the exemplary interaction and conceivable databases, is displayed (DP5) (Figure 3 right side). The process model with BPMN shows the individual steps of the initial service encounter and service recovery (see Figure 4). In line with the DPs, the customer has the option to abort the CA interaction and request a handover throughout the process (DP3). The information acquired from the customer (DP1 \& 2 ) is aggregated and supplemented with database entries to collect information for the handover (DP5). The handover is actively initiated, if more than three unfitting solutions have been proposed by the CA (DP4).

In order to verify the validity of the DPs, experts in customer service request handling watched the exemplary dialog, assessed the extracted information and depiction of the process. In general, the experts evaluated the instantiated DPs as suitable and applicable. Concerning DP1, the prototype showed an exemplary level of speech comprehension and dialog flow, which was rated as accurate and adequate. For the systematic collection of information (DP2), the experts considered the procedure of specifying and identifying a category of an incoming inquiry as efficient and appropriate. With regard to DP3, the experts agreed that the CA has to maintain the interaction with customers in order to extract substantive information from the conversation before the handover. The ability and procedure of the CA to actively initiate handovers were addressed regarding DP4. Experts stated that the faster a handover is triggered in the event of problems, the better. In accordance with the prototype, it was confirmed that the number of proposed unsuitable solutions by the CA constitutes a functional threshold for the initiation of a handover. Lastly, the experts evaluated the compilation and visualization of information. The prototype displayed following items: identification of the inquirer and/or object of inquiry, inquiry category and content, number of processing attempts, recommendation for continuation, keywords and conversation, historical database entries about the inquirer and/or object of inquiry. These items were rated to be both useful and helpful to instantly proceed after the handover (DP5).

\subsection{Expert assessment}

The experts considered the process sequence to be useful and practicable in order to facilitate HSAs in processing an inquiry after a real-time handover from a CA. In accordance to the prototypical CA interaction that manifest the modelled process steps, the experts validated the interdependence of CAs' information collection activities and information presentation for the process continuation by HSAs. Regarding DP1, the experts emphasized the importance of a solid understanding of language so that a request can be partially prepared for HSAs before handover. The CA's approach to systematically collect information was discussed to address DP2. Experts perceived the ability of the CA to identify the category of an inquiry as highly relevant. This capability enables the CA to subsequently pose relevant inquiry-specific questions. One expert expressed: "It makes sense to approach the problem step by step. If the CA knows what the inquiry is about, it can ask specific questions. Often only then you get the really important information." As a limitation, it was mentioned that inquiries can be ambiguous comprising several intentions, which complicates definite category allocation. As a result, a CA could develop the dialogue in the wrong direction from the start and incorrectly classify information. Therefore, CAs' capability for adaptive interaction and reassurance regarding customers' intentions is important in order to gather information through suitable questions. With regard to DP3, transparency 

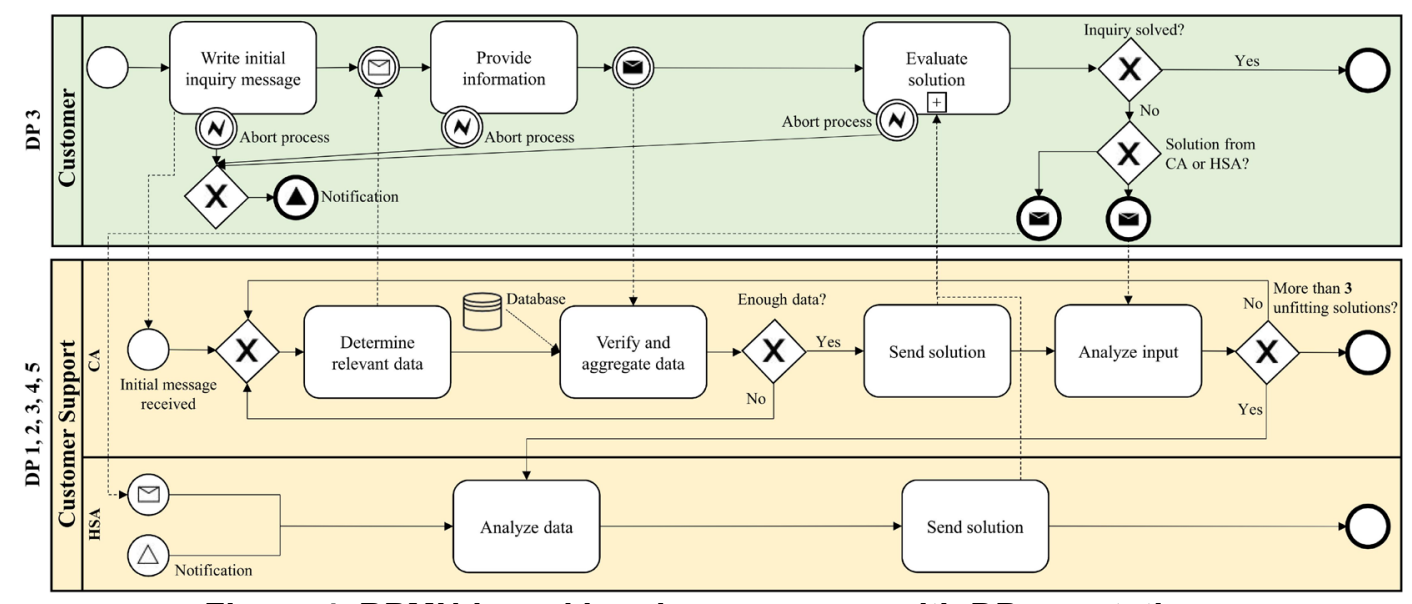

Figure 4. BPMN-based handover process with DP annotations

of behavior in terms of CA's feedback to customers concerning input processing problems is considered crucial to support customers' technology acceptance. One of the experts stated: the CA should "describe, [...] in which situation it is currently, whether it can proceed solving the problem or still has problems." The CA's request of customers to supplement or reformulate their input is helpful for maintaining the conversation. However, despite the relevance of transparent behavior, the CA should not repeatedly explain its shortcomings in detail to avoid customer frustration. The experts consider the option for customers to request a HSA, regardless of the service process stage, as mandatory to avoid customers' feeling of being dependent on the CA. In contrast to the presented process steps and prototype, one expert emphasized that customers who request a handover should be obliged to provide a minimum set of information (e.g. process number) to facilitate HSAs in continuing the inquiry processing and save valuable time. In addition, experts emphasized that structural changes need to be implemented to avoid overstressed HSAs and excessive rates of requested handovers from customers. Accordingly, one expert expressed that "if capacities are limited, it makes sense not to offer the possibility of a handover from the beginning, as otherwise the employees could be strained." The approach of CAs' initiation of handovers was discussed with respect to DP4. For the evaluated instantiation, the threshold was set at three failed attempts before the CA triggered the handover. This number was rated to be too high by two experts considering customers' patience. The specification of the threshold was determined to be use-case- and customer-specific and should not merely base on "human-oriented experience values" regarding inquiry processing standards. The exceedance of time limits as a threshold for handover initiation was considered as inappropriate, because the duration for an initial service encounter depends on customers' ability to engage with technology and communicate their intention. Regarding the format and types of information for the handover (DP5), the experts considered the presentation as valuable. One expert said that the "information was presented in a well-structured way". For the majority of experts, the most important information constitutes the identification of the inquirer and/or object and the content of the request. Particularly, two experts positively evaluated the summary of the conversation with keywords. The information presentation was assessed to facilitate the continuation of inquiry processing, as it allows more targeted questions and time saving searching for suitable information. The preparation of database entries is useful and offers the possibility to personalize the interaction with the customer. However, the overall applicability of database entries depends on the usage context and suitability of displaying historical data.

\section{Discussion and conclusion}

The objective of the paper was to develop a hybrid service recovery strategy for CAs in customer service. In line with companies' endeavor to increase efficiency of customer service with self-service technology by simultaneously reducing customers' dissatisfaction with CAs [1,9], we present initial design knowledge for real-time handovers to HSAs, which are initiated before CAs' service failure. The prescriptive design knowledge provides a solution to effectively and instantly escalate requests, if (1) CA-initiated repair attempts in collaboration with customers have failed or (2) they require HSA handling (e.g. due to company policies). The derived DPs embrace resource-friendly inquiry processing by considering, both, requirements for CA-customer interaction and the compilation of suitable information concerning the handover. 
The results of the evaluation confirm the suitability of the applied DPs in the instantiated prototype and process model. Overall, experts stated a positive perception toward the hybrid approach to execute service recoveries. They affirmed that efficient handovers require a concise compilation of critical and manageable information from the customer-CA interaction and associated databases for a seamless continuation of inquiry processing. The evaluation has further shown that the proposed process steps are applicable. However, the implementation requires a substantial restructuring of service processes to ensure the availability of HSAs for real-time handovers, while taking into account their time and mental capacities. Experts further emphasized the importance of a contextspecific determination of parameters and information items for the handover. Thus, the threshold for proactive handover initiations by CAs should comprise multiple data points from the customer-CA interaction covering aspects of time and CAs' solution or repair attempts.

With the presented MRs, DPs and instantiations, theoretical and practical contributions are provided. We contribute prescriptive knowledge about form and function with an initial set of design principles for research to guide the interlocking of CAs and HSAs to improve service recoveries, which incorporate request escalation [13, 19]. We also contribute to service literature by addressing the identified need in literature for improved service recovery strategies for CAs involving HSAs [12, 13, 17, 32, 38]. Established fallbacks involving request escalation are extended by providing a holistic service recovery strategy for CAs, addressing the challenge to provide real-time support and incorporating valuable insights from theory and experts in the field of customer service. With regard to practice, the DPs constitute a deployable design blueprint for organizations that aim to implement or improve service recovery strategies for CAs. This can serve to invoke, both, short resolution time for customers and support for HSAs to seamlessly continue processing inquiries after the handover. Apart from the promising results, there are some limitations to consider. The gathering of practical MRs and evaluation are limited to two independent samples of experts with a restricted demographic diversity. The insights are confined to their experience in handling inquiries representing customer-company encounters. However, following an iterative approach, further instantiations in future design cycles, will potentially generate supplementary practical knowledge. To enrich the nascent state of scientific knowledge, in future research the presented solution should be investigated in the application context and compared with existing market solutions that facilitate handovers from CAs to HSAs. Furthermore, the DPs should be implemented in a functional prototype to gain further insights about the applicability of the process steps and improvements of the artefact to the operational environment. Perceptions of employees regarding the usefulness of presented information need to be investigated in real-world usage scenarios. In this regard, mechanisms of an alert system should be developed and investigated addressing aspects of time and function to warn employees about upcoming handovers. The perspective of customers should also be addressed to assess the effect of real-time handovers on their satisfaction. Furthermore, future research should address the restructuring of service processes to integrate multiple service interfaces (CA and HSA) for effective service delivery and recovery. Lastly, the generalizability of the design should be validated for related service contexts such as intraorganizational service encounters (e.g. IT-helpdesk).

\section{Acknowledgements}

The research was financed with funding provided by the German Federal Ministry of Education and Research and the European Social Fund under the "Future of work" program (INSTANT, 02L18A110ff.).

\section{References}

[1] Barrett, M., E. Davidson, J. Prabhu, and S.L. Vargo, "Service Innovation in the Digital Age: Key Contributions and Future Directions", MIS Quarterly, 39(1), 2015, pp. 135-154.

[2] https://www.mckinsey.com/businessfunctions/operations/our-insights/service-innovationin-a-digital-world\#, accessed 7-8-2020.

[3] Meuter, M.L., A.L. Ostrom, R.I. Roundtree, and M.J. Bitner, "Self-Service Technologies: Understanding Customer Satisfaction with Technology-Based Service Encounters", Journal of Marketing, 64(3), 2000, pp. 50-64.

[4] https://www.gartner.com/en/newsroom/pressreleases/2018-02-19-gartner-says-25-percent-ofcustomer-service-operations-will-use-virtualcustomer-assistants-by-2020, accessed 7-8-2020.

[5] McTear, M., Z. Callejas, and D. Griol, The Conversational Interface: Talking to Smart Devices, Springer, Cham, 2016.

[6] Schuetzler, R.M., J.S. Giboney, G.M. Grimes, and J.F. Nunamaker, "The Influence of Conversational Agent Embodiment and Conversational Relevance on Socially Desirable Responding", Decision Support Systems, 114, 2018, pp. 94-102.

[7] https://www.ibm.com/blogs/watson/2017/10/howchatbots-reduce-customer-service-costs-by-30percent/, accessed 7-8-2020. 
[8] Marinova, D., K. de Ruyter, M.-H. Huang, M.L. Meuter, and G. Challagalla, "Getting Smart: Learning From Technology-Empowered Frontline Interactions", Journal of Service Research, 20(1), 2017, pp. 29-42.

[9] Verhagen, T., J. van Nes, F. Feldberg, and W. van Dolen, "Virtual Customer Service Agents: Using Social Presence and Personalization to Shape Online Service Encounters", Journal of Computer-Mediated Communication, 19(3), 2014, pp. 529-545.

[10] Waizenegger, L., I. Seeber, G. Dawson, and K. Desouza, "Conversational Agents - Exploring Generative Mechanisms and Second-hand Effects of Actualized Technology Affordances", in 53rd Hawaii International Conference on System Sciences (HICSS). 2020: Wailea, USA.

[11] Kvale, K., O.A. Sell, S. Hodnebrog, and A. Følstad, "Improving Conversations: Lessons Learnt from Manual Analysis of Chatbot Dialogues", in Chatbot Research and Design, A. Følstad, T. Araujo, S. Papadopoulos, E.L.-C. Law, O.-C. Granmo, E. Luger, and P.B. Brandtzaeg, Editors. 2020. Springer International Publishing: Cham.

[12] Grudin, J. and R. Jacques, "Chatbots, Humbots, and the Quest for Artificial General Intelligence", in 2019 CHI Conference on Human Factors in Computing Systems. 2019: Glasgow, Scotland.

[13] Corea, C., P. Delfmann, and S. Nagel, "Towards Intelligent Chatbots for Customer Care - PracticeBased Requirements for a Research Agenda", in 53rd Hawaii International Conference on System Sciences (HICSS). 2020: Wailea, USA.

[14] Collier, J.E., M. Breazeale, and A. White, "Giving Back the "Self" in Self Service: Customer Preferences in Self-service Failure Recovery", Journal of Services Marketing, 31(6), 2017, pp. 604-617.

[15] Bock, D.E., J.S. Wolter, and O.C. Ferrell, "Artificial Intelligence: Disrupting What We Know About Services", Journal of Services Marketing, 34(3), 2020, pp. 317-334.

[16] Lu, V.N., J. Wirtz, W.H. Kunz, S. Paluch, T. Gruber, A. Martins, and P.G. Patterson, "Service Robots, Customers and Service Employees: What Can We Learn from the Academic Literature and Where are the Gaps?", Journal of Service Theory and Practice, 30(3), 2020, pp. 361-391.

[17] Castillo, D., A.I. Canhoto, and E. Said, "The Dark Side of AI-powered Service Interactions: Exploring the Process of Co-destruction from the Customer Perspective", The Service Industries Journal, 2020, pp. 1-26.

[18] Følstad, A. and C. Taylor, "Conversational Repair in Chatbots for Customer Service: The Effect of Expressing Uncertainty and Suggesting Alternatives", in Chatbot Research and Design, A. Følstad, T. Araujo, S. Papadopoulos, E.L.-C. Law, O.-C. Granmo, E. Luger, and P.B. Brandtzaeg, Editors. 2020. Springer International Publishing: Cham.

[19] Kucherbaev, P., A. Bozzon, and G.-J. Houben, "Human-Aided Bots", IEEE Internet Computing, 22(6), 2018, pp. 36-43.
[20] Ashktorab, Z., M. Jain, Q.V. Liao, and J.D. Weisz, "Resilient Chatbots: Repair Strategy Preferences for Conversational Breakdowns", in 2019 CHI Conference on Human Factors in Computing Systems. 2019: Glasgow, Scotland.

[21] Vom Brocke, J., W. Maaß, P. Buxmann, A. Maedche, J.M. Leimeister, and G. Pecht, "Future Work and Enterprise Systems", Business \& Information Systems Engineering, 60(4), 2018, pp. 357-366.

[22] Alter, S., "Work System Theory: Overview of Core Concepts, Extensions, and Challenges for the Future", Journal of the Association for Information Systems, 14(2), 2013, pp. 72-121.

[23] Seeber, I., E. Bittner, R.O. Briggs, T. de Vreede, G.-J. de Vreede, A. Elkins, R. Maier, A.B. Merz, S. OesteReiß, N. Randrup, G. Schwabe, and M. Söllner, "Machines as Teammates: A Research Agenda on AI in Team Collaboration", Information \& Management, 57(2), 2020, p. 103174.

[24] Wilson, H.J. and P.R. Daugherty, "Collaborative Intelligence: Humans and AI are Joining Forces", Havard Business Review, 96(July-August), 2018, pp. 115-123.

[25] Seeber, I., L. Waizenegger, S. Seidel, S. Morana, I. Benbasat, and P.B. Lowry, "Collaborating with Technology-based Autonomous Agents", Internet Research, 2020.

[26] Bittner, E.A.C., S. Oeste-Reiß, and J.M. Leimeister, "Where is the Bot in our Team? Toward a Taxonomy of Design Option Combinations for Conversational Agents in Collaborative Work", in 52nd Hawaii International Conference on System Sciences (HICSS). 2019: Grand Wailea, USA.

[27] Diederich, S. and A.B. Brendel, "On Conversational Agents in Information Systems Research: Analyzing the Past to Guide Future Work", in 14th International Conference on Wirtschaftsinformatik. 2019: Siegen, Germany.

[28] Gnewuch, U., S. Morana, and A. Maedche, "Towards Designing Cooperative and Social Conversational Agents for Customer Service", in 38th International Conference on Information Systems (ICIS). 2017: Seoul, South Korea.

[29] Weizenbaum, J., "ELIZA- A Computer Program for the Study of Natural Language Communication between Man and Machine", Communications of the ACM, 9(1), 1966, pp. 36-45.

[30] Knijnenburg, B.P. and M.C. Willemsen, "Inferring Capabilities of Intelligent Agents from Their External Traits", ACM Transactions on Interactive Intelligent Systems, 6(4), 2016, pp. 1-25.

[31] Meyer von Wolff, R., S. Hobert, and M. Schumann, "How May I Help You? - State of the Art and Open Research Questions for Chatbots at the Digital Workplace", in 52nd Hawaii International Conference on System Sciences (HICSS). 2019: Grand Wailea, USA.

[32] Følstad, A. and M. Skjuve, "Chatbots for Customer Service: User Experience and Motivation", in 1st International Conference on Conversational User Interfaces. 2019: Dublin, Ireland. 
[33] Keyser, A. de, S. Köcher, L. Alkire, C. Verbeeck, and J. Kandampully, "Frontline Service Technology Infusion: Conceptual Archetypes and Future Research Directions", Journal of Service Management, 30(1), 2019, pp. 156-183.

[34] Diederich, S., T.-B. Lembcke, A.B. Brendel, and L.M. Kolbe, "Not Human After All: Exploring the Impact of Response Failure on User Perception of Anthropomorphic Conversational Service Agents", in 28th European Conference on Information Systems (ECIS). 2020: Marrakesh, Marocco.

[35] Luger, E. and A. Sellen, "Like Having a Really Bad PA: The Gulf between User Expectation and Experience of Conversational Agents", in 2016 CHI Conference on Human Factors in Computing Systems. 2016: San Jose, USA.

[36] Kocielnik, R., S. Amershi, and P.N. Bennett, "Will You Accept an Imperfect AI?", in 2019 CHI Conference on Human Factors in Computing Systems. 2019: Glasgow, Scotland.

[37] Luo, X., Z. Lin, and Wang, Y., Nie, Z., "CoChat: Enabling Bot and Human Collaboration for Task Completion", in 13th AAAI Conference on Artificial Intelligence. 2018.

[38] Feine, J., S. Morana, and U. Gnewuch, "Measuring Service Encounter Satisfaction with Customer Service Chatbots Using Sentiment Analysis", in 14th International Conference on Wirtschaftsinformatik. 2019: Siegen, Germany.

[39] Bitner, M.J. and H.S. Wang, "Service Encounters in Service Marketing Research", in Handbook of Service Marketing Research, R. Rust and M.-H. Huang, Editors. 2014. Edward Elgar Publishing.

[40] Scherer, A., N.V. Wünderlich, and F. von Wangenheim, "The Value of Self-Service: Long-Term Effects of Technology-Based Self-Service Usage on Customer Retention", MIS Quarterly, 39(1), 2015, pp. 177-200.

[41] Patrício, L., R.P. Fisk, and J. Falcão e Cunha, "Designing Multi-Interface Service Experiences: The Service Experience Blueprint", Journal of Service Research, 10(4), 2008, pp. 318-334.

[42] McLean, G. and K. Osei-Frimpong, "Examining Satisfaction with the Experience during a Live Chat Service Encounter-Implications for Website Providers", Computers in Human Behavior, 76, 2017, pp. 494-508.

[43] Larivière, B., D. Bowen, T.W. Andreassen, W. Kunz, N.J. Sirianni, C. Voss, N.V. Wünderlich, and A. de Keyser, "'Service Encounter 2.0": An Investigation into the Roles of Technology, Employees and Customers", Journal of Business Research, 79, 2017, pp. 238-246.

[44] Diederich, S., M. Janßen-Müller, A.B. Brendel, and S. Morana, "Emulating Empathetic Behavior in Online Service Encounters with Sentiment-Adaptive Responses: Insights from an Experiment with a Conversational Agent", in 40th International Conference on Information Systems (ICIS). 2019: München, Germany.

[45] Bitner, M.J., S.W. Brown, and M.L. Meuter, "Technology Infusion in Service Encounters", Journal of the Academy of Marketing Science, 28(1), 2000, pp. 138-149.

[46] Spreng, R.A., G.D. Harrell, and R.D. Mackoy, "Service Recovery: Impact on Satisfaction and Intentions", Journal of Services Marketing, 9(1), 1995, pp. 15-23.

[47] Prasongsukarn, K. and P.G. Patterson, "An Extended Service Recovery Model: The Moderating Impact of Temporal Sequence of Events", Journal of Services Marketing, 26(7), 2012, pp. 510-520.

[48] Hevner, Alan, R., "A Three Cycle View of Design Science Research", Scandinavian Journal of Information Systems, 19(2), 2007, pp. 87-92.

[49] Myers, M.D. and M. Newman, "The Qualitative Interview in IS Research: Examining the Craft", Information and Organization, 17(1), 2007, pp. 2-26.

[50] Mayring, P., Qualitative Content Analysis: Theoretical Foundation, Basic Procedures and Software Solution, 2014.

[51] Chandra, L., S. Seidel, and S. Gregor, "Prescriptive Knowledge in IS Research: Conceptualizing Design Principles in Terms of Materiality, Action, and Boundary Conditions", in 48th Hawaii International Conference on System Sciences (HICSS). 2015: Kauai, USA.

[52] Gregor, S., "The Nature of Theory in Information Systems", 30(3), 2006, pp. 611-642.

[53] Bitner, M.J., B.H. Booms, and M.S. Tetreault, "The Service Encounter: Diagnosing Favorable and Unfavorable Incidents", Journal of Marketing, 54(1), 1990, pp. 71-84.

[54] Schuetzler, R.M., G.M. Grimes, J.S. Giboney, and J. Buckman, "Facilitating Natural Conversational Agent Interactions: Lessons from a Deception Experiment", in 35 th International Conference on Information Systems (ICIS). 2014: Auckland, New Zealand.

[55] Følstad, A., C.B. Nordheim, and C.A. Bjørkli, "What Makes Users Trust a Chatbot for Customer Service? An Exploratory Interview Study", in Internet Science, S.S. Bodrunova, Editor. 2018. Springer International Publishing: Cham.

[56] Reshmi, S. and K. Balakrishnan, "Implementation of an Inquisitive Chatbot for Database Supported Knowledge Bases", Sādhanā, 41(10), 2016, pp. 1173-1178.

[57] Zeithaml, V.A., A. Parasuraman, and L.L. Berry, "Problems and Strategies in Services Marketing", Journal of Marketing, 49(2), 1985, p. 33.

[58] Zumstein, D. and S. Hundertmark, "Chatbots - An Interactive Technology for Personalized Communication, Transactions and Services", IADIS International Journal on WWW/Internet, 15(1), 2017, pp. 96-109.

[59] Jones, D. and S. Gregor, "The Anatomy of a Design Theory", Journal of the Association for Information Systems, 8(5), 2007, pp. 312-335.

[60] Möller, F., T. Guggenberger, and B. Otto, "Towards a Method for Design Principle Development in Information Systems", in 15th International Conference on Design Science Research in Information Systems and Technology (DESRIST). 2020: Kristiansand, Norway. 